\title{
Genome-wide SNPs lead to strong signals of geographic structure and relatedness patterns in the major arbovirus vector, Aedes aegypti
}

Gordana Rašić, Igor Filipović, Andrew R Weeks and Ary A Hoffmann

\begin{abstract}
Background: Genetic markers are widely used to understand the biology and population dynamics of disease vectors, but often markers are limited in the resolution they provide. In particular, the delineation of population structure, fine scale movement and patterns of relatedness are often obscured unless numerous markers are available. To address this issue in the major arbovirus vector, the yellow fever mosquito (Aedes aegypti), we used double digest Restriction-site Associated DNA (ddRAD) sequencing for the discovery of genome-wide single nucleotide polymorphisms (SNPs). We aimed to characterize the new SNP set and to test the resolution against previously described microsatellite markers in detecting broad and fine-scale genetic patterns in Ae. aegypti.

Results: We developed bioinformatics tools that support the customization of restriction enzyme-based protocols for SNP discovery. We showed that our approach for RAD library construction achieves unbiased genome representation that reflects true evolutionary processes. In Ae. aegypti samples from three continents we identified more than 18,000 putative SNPs. They were widely distributed across the three Ae. aegypti chromosomes, with $47.9 \%$ found in intergenic regions and $17.8 \%$ in exons of over 2,300 genes. Pattern of their imputed effects in ORFs and UTRs were consistent with those found in a recent transcriptome study. We demonstrated that individual mosquitoes from Indonesia, Australia, Vietnam and Brazil can be assigned with a very high degree of confidence to their region of origin using a large SNP panel. We also showed that familial relatedness of samples from a $0.4 \mathrm{~km}^{2}$ area could be confidently established with a subset of SNPs.

Conclusions: Using a cost-effective customized RAD sequencing approach supported by our bioinformatics tools, we characterized over 18,000 SNPs in field samples of the dengue fever mosquito Ae. aegypti. The variants were annotated and positioned onto the three Ae. aegypti chromosomes. The new SNP set provided much greater resolution in detecting population structure and estimating fine-scale relatedness than a set of polymorphic microsatellites. RAD-based markers demonstrate great potential to advance our understanding of mosquito population processes, critical for implementing new control measures against this major disease vector.
\end{abstract}

Keywords: Aedes aegypti, Restriction-site associated DNA sequencing, In silico genome digestion, Fastq file demultiplexing, Genome-wide single nucleotide polymorphisms, Mosquito population genomics

\footnotetext{
*Correspondence: gordana.rasic@unimelb.edu.au

Pest and Disease Vector Group, Department of Genetics, The University of Melbourne, Victoria 3010, Australia
}

\section{Biomed Central}

(c) 2014 Rašić et al.; licensee BioMed Central Ltd. This is an Open Access article distributed under the terms of the Creative Commons Attribution License (http://creativecommons.org/licenses/by/2.0), which permits unrestricted use, distribution, and reproduction in any medium, provided the original work is properly credited. The Creative Commons Public Domain Dedication waiver (http://creativecommons.org/publicdomain/zero/1.0/) applies to the data made available in this article unless otherwise stated. 


\section{Background}

The yellow fever mosquito, Aedes aegypti (Culicidae, Diptera), is the major vector of human arboviruses. Because of its tight connection to humans and its distribution in most sub-tropical and tropical regions, this insect causes a substantial burden on global public health [1]. The development of anti-viral vaccines has been largely unsuccessful and has therefore shifted focus in disease prevention back to the control of Ae. aegypti populations [2]. Understanding basic ecological and microevolutionary processes in mosquito populations is necessary for the efficient implementation of many control measures. For example, knowledge of the rates of ongoing gene flow, fine scale mosquito movement and adaptive genomic changes are important for predicting the spread of Wolbachia infection $[3,4]$ or insecticide resistance [5].

Ecological processes can often be inferred indirectly from observed genetic patterns [6] and this has led to interest in developing molecular markers for population studies in Ae. aegypti. In the last decade, mitochondrial DNA (mtDNA) sequences have commonly been used for Ae aegypti population genetic studies [7]. However, mtDNA markers are best suited for analyses of historical processes at larger geographic scales, as they can only provide information on long-term accumulated effects of female dispersal due to their moderate mutation rate and maternal mode of inheritance [8]. Furthermore, the recent discovery of mtDNA pseudogenes (Numts) in the nuclear genome of Ae. aegypti limits the reliability of inferences from mtDNA [9]. Fast-mutating, highly variable nuclear markers such as microsatellites enable efficient analyses of fine-scale contemporary processes [8]. Close association with repetitive genetic elements and lower polymorphism has hindered the development of a robust set of microsatellite markers in Ae. aegypti until the complete genome sequence became available [10-12]. Population genetic structure of this vector has since been usually ascertained with up to 12 microsatellite loci [13], that were sometimes supplemented with a few single nucleotide polymorphism (SNP) markers [14] or ExonPrimed Intron Crossing (EPIC) markers [15-17].

Based on these marker systems, some details of Ae. aegypti movement and gene flow patterns have been inferred. Studies have investigated genetic structure across different spatial scales $[7,13]$, and some have also included a temporal component [15-17]. Despite reports of limited active dispersal of this mosquito [18], a high level of gene flow has often been found even at broader regional and continental scales $[15,19]$. Unexpected structure may be explained by passive, human-mediated dispersal of mosquito eggs, larvae and adults coupled with low migration rates $[7,13,20]$. However, methodological issues associated with the available genetic markers $[6,8]$ remain a major obstacle in using these markers to understand population movement patterns and microspatial scale structuring across time.

Next generation sequencing provides an opportunity to generate SNP markers at a genome-wide level even for non-model species. A mere 50 bi-allelic SNPs can provide the same resolution as 20 highly variable microsatellites for distinguishing closely related individuals [21]. A large panel of SNPs should therefore detect weak genetic structure caused by recent ecological and evolutionary processes [8] and provide reliable inferences of demographic history [22]. Genome-wide SNPs would thus be ideal for determining patterns of dispersal, gene flow and genetic structure at all spatial scales for populations of Ae. aegypti.

An analysis of genome-wide sequence variation via whole genome sequencing is still prohibitively expensive for Ae. aegypti, given its large genome size (1.3 Gbp; [23]). A cost-effective alternative is to sequence a fraction of the genome through Restriction-site Associated DNA sequencing (RADseq) [24,25]. RAD sequencing has been successfully used for the discovery of thousands of markers in yeast, plants and animals [26,27]. RAD loci are DNA fragments adjacent to the cut site of a particular restriction enzyme, or a combination of two enzymes [28]. Choice of restriction enzyme(s) (e.g. frequent or infrequent cutters) and the fragment size selection window can be used to optimize the number of tags, capturing a certain proportion of the genome for SNP detection and comparison across multiple individuals or populations.

Recently, Brown et al. [29] used 1,503 SNPs generated with RAD sequencing to test the hypothesis about global invasion and domestication of Aedes aegypti. Here, we developed bioinformatics tools that support the customization of restriction-enzyme-based protocols and used them to discover over 18,000 SNPs in Ae. aegypti collected on three continents. We have positioned these SNPs onto the newly assembled Ae. aegypti reference genome [30] and provided their annotation and prediction of effects. We tested the resolution of these new markers against a set of previously isolated microsatellites in delineating neotropical populations and establishing relatedness patterns at a fine spatial scale. Overall, we demonstrated the high potential of genome-wide SNP markers to advance our understanding of mosquito population dynamics which is crucial for improving the control measures against this major disease vector.

\section{Results and discussion}

Bioinformatics tools: DDsilico and DDemux

We developed DDsilico as a memory efficient program written in $\mathrm{C}$ language for in silico genome digestion with a single restriction enzyme or a combination of enzymes. This program calculates the number of potential RAD loci from the available genome sequence (see Additional file 1). 
DDsilico can be used for any restriction-enzyme-based protocol and is particularly suitable for customizing the double digest (dd)RADseq protocol [28] because it separates fragments that have different overhangs on two ends (i.e. potential double digest RAD loci) from nonusable fragments. We tested the performance of DDsilico by comparing results with the Bioanalyzer profiles of empirical digestions. DNA of the transformation vector Stinger GFP was digested with restriction enzymes NlaIII and $M l u C I$ (NEB). Observed and expected fragment profiles were highly concordant for sizes over $150 \mathrm{bp}$ (see Additional file 2). Due to the purification of digestion reactions required for the Bioanalyzer run, fragments smaller than 150 bp were only partially retained with the paramagnetic bead solution.

To estimate the number of potential ddRAD tags in the Ae. aegypti genome, we performed in silico double digestion with various combinations of restriction enzymes. Distinguishing between usable fragments is a feature of DDsilico, as some double digestions produce numerous fragments in the desirable size range (100-500 bp), but only a small proportion constitutes potential ddRAD loci (see Additional file 3). Based on in silico results, we chose two frequent cutter enzymes (NlaIII and MluCI) for our ddRAD library construction. The ratio between the number of loci in the Ae. aegypti catalog $(574,715)$ and the number of loci predicted with DDsilico $(641,234)$ was $89.6 \%$.

DDsilico can be used for any method that utilizes restriction enzyme(s) to create libraries with a reduced genome representation, such as Genotyping-by-sequencing (GBS) [31,32], Reduced-representation bi-sulfate sequencing (RRBS) [33], ezRAD [34] or RESTseq [35]. Simulating genome digestions enables researchers to choose optimal enzymes for specific species and research questions. For example, using rare cutting enzymes allows for higher level of sample multiplexing but can result in a non-uniform complexity reduction and biased distribution of sequenced fragments [35], leading to inaccurate estimation of population parameters or average methylation levels [33].

We also developed DDemux as a demultiplexer of fastq files under various barcoding schemes (see Additional file 4). Our program is memory efficient, developed in $\mathrm{C}$ language and easy to execute on Windows and Ubuntu platforms. It supports samples labelled with one or two barcodes that can vary in length. DDemux can use barcode sequences found in P1 and/or P2 reads, and in the Index reads (Illumina sequencing platforms). In our library, each sample was defined by a combination of variable length barcodes at the 5' and 3' ends (found in $\mathrm{P} 1$ and $\mathrm{P} 2$ reads). Such a combinatorial scheme reduces the cost for generating adapters with unique barcodes, while the varying length of barcodes increases library diversity at the 5' and 3' ends. This is particularly useful for ddRAD and other non-shearing-based libraries, where paired end (P2) reads can be used for SNP discovery but lack any diversity in the initial sequencing cycles when barcodes are absent.

We developed a customized pipeline that takes individually sorted raw sequences and outputs files ready for SNP calling (Figure 1, see Additional file 5). It automates standard processes for read quality control and aligning to a reference genome. Following demultiplexing, sequence quality scores are automatically converted into the Sanger format. This ensures standardization of quality scores for data generated by sequencing machines with different QC set-ups. Single (P1) and paired (P2) reads are then filtered and trimmed according to the chosen Phred score with FASTX-Toolkit (http://hannonlab.cshl.edu/fastx_toolkit/). P1 and P2 reads are then matched, leaving the unpaired reads as orphans. Paired reads are first aligned to the reference genome using Bowtie [36] according to the selected parameters. User can choose to also perform single-end alignment using joined orphans and all unaligned paired reads. In the final step, all aligned output files are merged per individual and are ready for downstream analyses.

\section{Discovery and characterization of SNPs in Ae. aegypti}

Prior to any quality filtering, the average number of reads per individual was 3.31 million, ranging between 1.67 and 5.99 million reads. Quality filtering removed on average $5.5 \%$ of reads, retaining sequences with a mean quality score of 38 and a GC content of $41 \%$. On average, $72.7 \%$ high quality reads aligned uniquely to the Ae. aegypti reference genome. With a minimum depth of five reads per individual, aligned sequences formed 574,715 RAD loci in the catalogue created with the Stacks pipeline for SNP discovery [37].

We further filtered loci that were present in at least $75 \%$ of individuals and retained 13,591 loci with an average $12 \times$ read depth (Table 1). 9,611 loci (70.72\%) were polymorphic in at least one geographic sample, giving 18,147 putative biallelic SNPs (see Additional file 6). When we applied the same filtering criterion to each of the four samples, between 6,877 and 8,755 SNPs per sample were obtained. Number of private variants was the largest in the collection from Brazil $(2,511)$ and the lowest from Vietnam $(1,200)$. Average minor allele frequency per sample was between 0.187 (Vietnam) and 0.211 (Brazil) (Table 1).

Most common sequencing errors of the Illumina machines are $A \leftrightarrow C$ and $G \leftrightarrow T$ transversions [38]. In our filtered data-set, two thirds of SNPs were transitions (transition:transversion ratio 1.62), suggesting a very small influence of sequencing error on our SNP calling. This is highly comparable to other RADseq studies that reported 


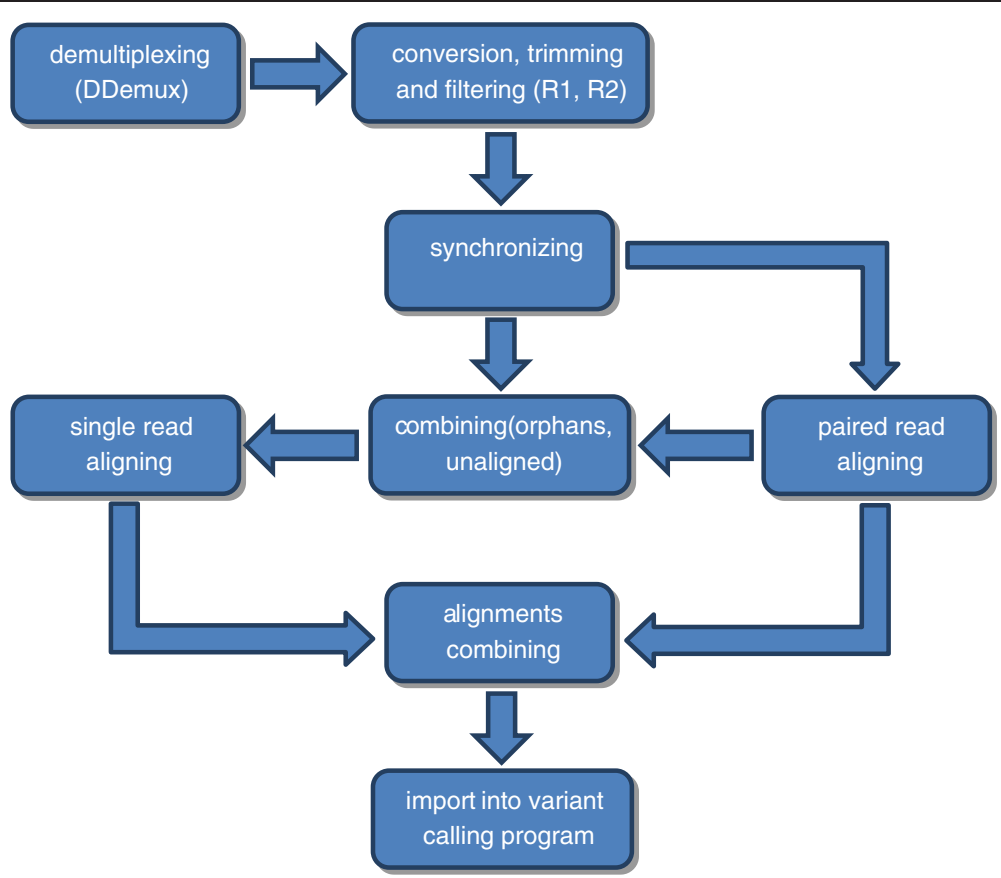

Figure 1 Schematic representation of the customized pipeline for automated sequence processing. After demultiplexing using DDemux, quality scores of individually sorted raw sequencing reads are converted to Sanger format. Reads are then filtered and trimmed according to the given parameters. Retained reads are then synchronyzed as pairs with identical coordinates, and unpaired reads sorted as orphans. Paired reads are aligned to the reference genome using Bowtie [36]. Unaligned and orphan reads are then joined, and user can choose to perform single read alignment. Finally, all aligned reads are joined and are ready for a variant calling pipeline.

a ratio of 1.6 in the European eel [39], 1.65 in the eggplant [40] and 1.7 in the great tit [41].

SNPs from our catalog were distributed across 1,036 supercontigs that constitute $89 \%$ of the Ae. aegypti genome. Their number was significantly correlated with the size of supercontigs (Pearson $r=0.783, p<0.01$ ). Thanks to the recently improved assembly of the A. aegypti genome [30] we were able to position $66.4 \%$ of uncovered SNPs onto the three $A$. aegypti chromosomes: 2,423 SNPs on chromosome 1, 5,313 on chromosome 2 and 4,320 on chromosome 3 (see Additional file 6).

We found $47.9 \%$ of SNPs in intergenic regions, $17.7 \%$ in introns and $17.8 \%$ in exons of 2,374 genes (Figure 2). They had very low occurrence in splice-site donor sequences and UTR 5' region ( $0.035 \%$ and $0.301 \%$ respectively), and somewhat higher occurrence in UTR 3' region (1.31\%). Using the program SNPEff [42], we imputed the overall impact of all variants as largely modifying (82.1\%), followed by low $(11.7 \%)$, moderate $(5.82 \%)$ and high impact $(0.395 \%)$ (Figure 3, see Additional file 6).

Reduced representation libraries generally locate only around $2 \%$ of SNPs in the transcribed part of the genome [39], but they are generated using a rare cutting enzyme and random shearing. Here, we demonstrate the advantage of using two very frequent cutting enzymes that generate RAD loci evenly distributed across the genome. Other methods, such as RRSB, RESTseq or modified GBS, also utilize "common-cutters" to improve genome coverage and achieve unbiased distribution of sequenced loci $[32,33,35]$.

Table 1 Summary statistics for filtered RAD loci

\begin{tabular}{cccccccccccc}
\hline & $\boldsymbol{n}$ & $\mathbf{T}$ & \% pol & SNP & Private & $\boldsymbol{P}$ & $\boldsymbol{H}_{\mathrm{O}}$ & $\boldsymbol{H}_{\mathrm{E}}$ & $\boldsymbol{F}_{\text {IS }}$ & $\boldsymbol{\pi}$ & $\boldsymbol{N}_{\mathbf{e}}$ \\
\hline $\mathrm{Br}$ & 17 & 24273 & 23.64 & 8755 & 2511 & 0.800 & 0.265 & 0.282 & 0.079 & 0.0014 & 23083 \\
$\mathrm{Au}$ & 17 & 25019 & 21.68 & 8026 & 1310 & 0.810 & 0.234 & 0.268 & 0.120 & 0.0012 & 19479 \\
$\mathrm{In}$ & 13 & 25002 & 20.87 & 7757 & 1423 & 0.809 & 0.253 & 0.270 & 0.083 & 0.0012 & 19230 \\
$\mathrm{Vi}$ & 15 & 22333 & 20.61 & 6877 & 1200 & 0.815 & 0.243 & 0.261 & 0.079 & 0.0011 & 18401 \\
$\mathrm{All}$ & 62 & 13591 & 70.72 & 18147 & & & & & &
\end{tabular}

$n$ - the number of analyzed individuals from Brazil (Br), Australia (Au), Indonesia (In) and Vietnam (Vi); $\mathrm{T}$ - the number of RAD loci; \% pol - percentage of polymorphic loci; SNP - total number of SNPs; private - the number of private SNPs; $P$ - average frequency of the more common allele; $H_{\mathrm{O}}, H_{\mathrm{E}}-$ observed and expected heterozygosity at polymorphic sites; $F_{\mathrm{IS}}$ - fixation index across polymorphic sites; $\pi$ - average nucleotide diversity (calculated across polymorphic and non-variant sites); $N_{\mathrm{e}}-$ lower range effective population size (for $\mu=10^{-8}$ per site per generation). 


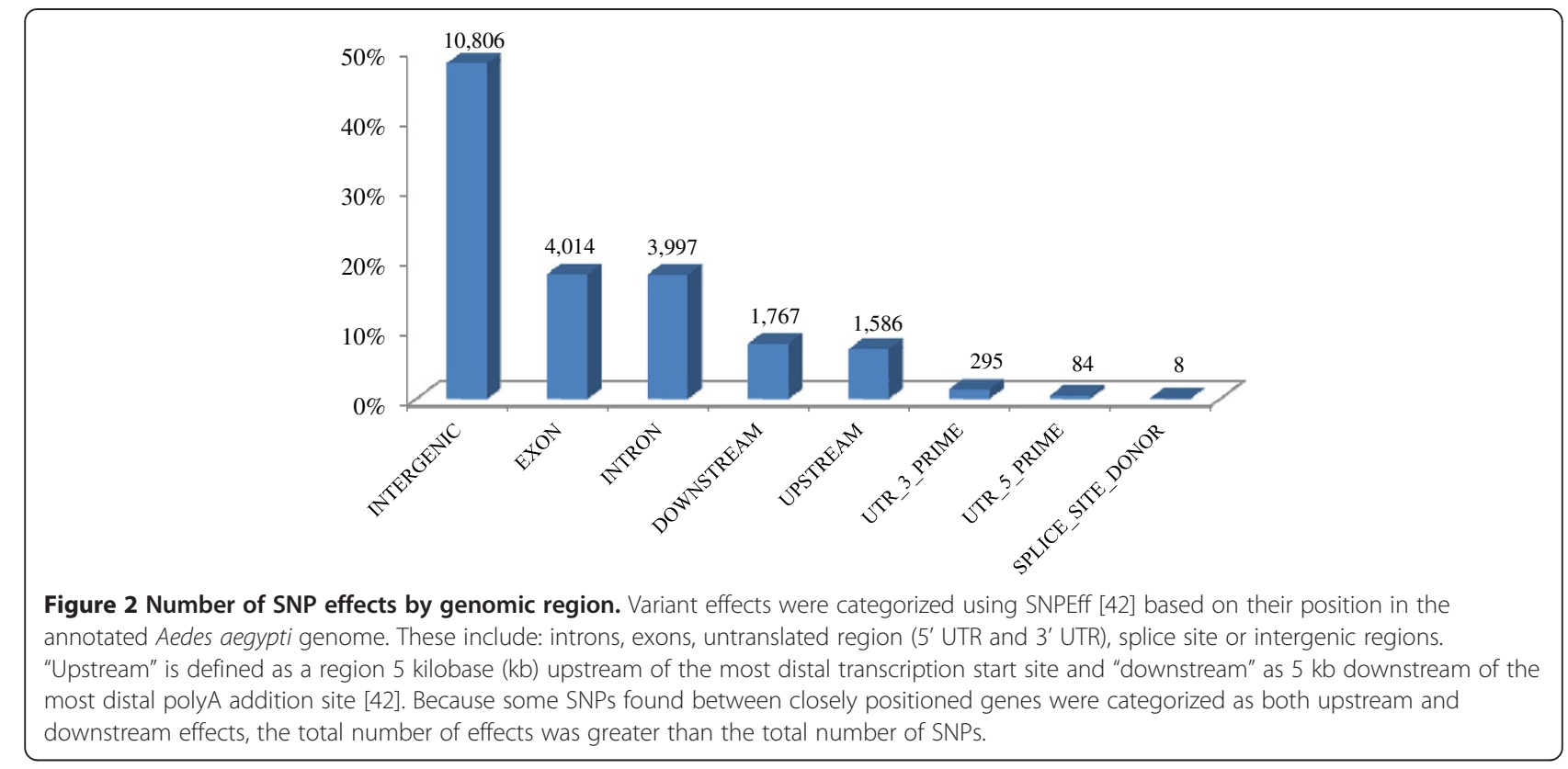

Recently, Bonizzoni et al. [43] used RNAseq to analyze sequence variation in the transcriptome of three Ae. aegypti laboratory strains. They characterized over 130,000 SNPs in open-reading frames (ORFs) and untranslated regions (UTRs) of 4,492 genes. In our catalog, $19 \%$ of all SNPs were found within ORFs and UTRs of 2,374 genes. As in Bonizzoni et al., the most common SNPs were synonymous, 3'UTRs contained 4 times more SNPs than 5'UTRs, and SNPs that affect splice site donors, start and stop codons were very rare $($ total $<1 \%)$. We uncovered more non-synonymous changes than the mentioned study, likely because we analyzed pantropical Ae. aegypti populations that are very divergent from the laboratory reference strain LVP $[13,29]$.

\section{Genetic diversity and effective population size}

Average observed heterozygosity per variable SNP site was lower (0.23-0.26) than the expected heterozygosity (0.27-0.28) in all samples, with overall $F_{\text {IS }}$ values ranging between 0.079 and 0.120 (Table 1). Microsatellite loci also showed lower than expected heterozygosity in all samples except for Vietnam, with $F_{\mathrm{IS}}$ ranging between 0.025 and 0.173 (see Additional file 7). Microsatellite loci were moderately polymorphic in all samples, with two to seven alleles per locus (average 3.75-4.50 alleles per locus).

Nucleotide diversity averaged over all SNP loci $(\pi)$ was lower and comparable among samples (0.0011-0.0014, Table 1). Due to numerous low frequency alleles, this

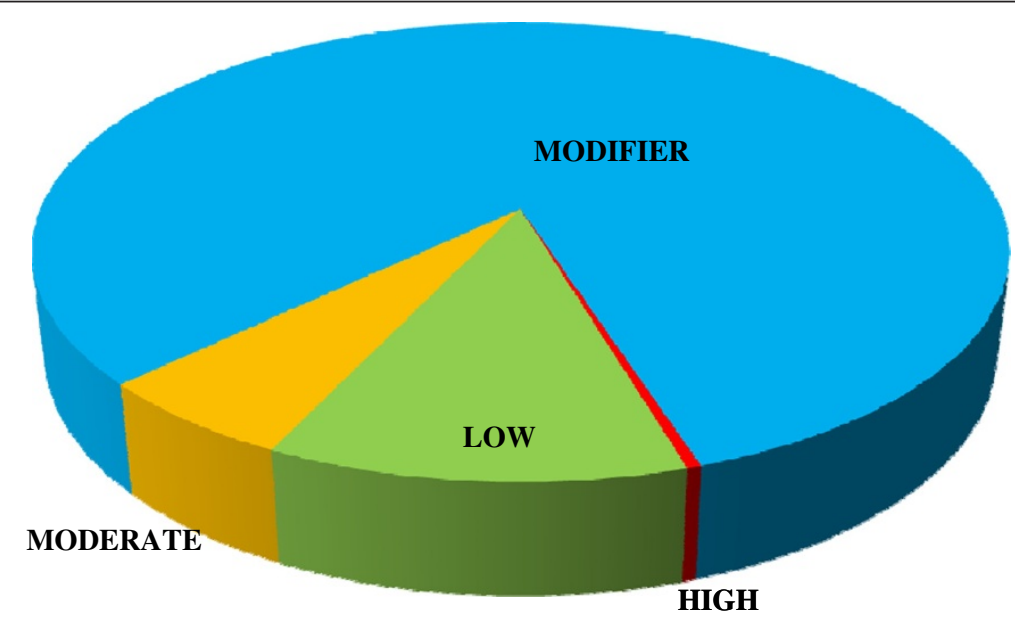

Figure 3 Number of SNP effects by impact. SNP effects were categorized by impact as high (affecting splice-sites, stop and start codons), moderate (non-synonymous), low (synonymous coding/start/stop, start gained), and modifier (upstream, downstream, intergenic, UTR). 
diversity index (equivalent to expected heterozygosity) was an order of magnitude lower than previously reported (e.g. 0.012 in [44]). Based on average nucleotide diversity, long term effective population sizes were estimated to range between 18,000 (for $\mu=10^{-8}$ per site per generation) and 230,000 (for $\mu=10^{-9}$ per site per generation) across four collections (Table 1). We note that SNPs may provide more biased estimates of long-term $N_{\mathrm{e}}$ than faster evolving markers such as microsatellites, because they are likely to be more affected by mutationdrift deviations [45]. However, our long term $N_{\mathrm{e}}$ based on the higher mutation rate $\left(10^{-8}\right.$ per site per generation) are concordant with the estimates of census population sizes at these collection sites. For example, Jeffery et al. [46] found that the number of Ae. aegypti adult females in Tri Nguyen village (Vietnam) could be as high as 26,431 individuals (95\% CI 15,474-37,489), while we found the lower range of $N_{\mathrm{e}}$ to be 18,401 individuals (Table 1). Based on Wolbachia-infected Ae. aegypti releases in Gordonvale (Australia), a recent estimate for adult females within the release area was 7,261 individuals [47]. Assuming equal sex ratio, total census size in
Gordonvale would be around 14,500 individuals, while our lower range $N_{\mathrm{e}}$ estimate was 19,479 individuals. Hence, we consider our SNP panel to be reflecting true evolutionary processes in Ae. aegypti populations.

\section{Using genome-wide SNPs to examine population processes \\ Broad-scale structuring}

Our genome-wide SNP set demonstrated high power in resolving Ae. aegypti genetic structure at a broad spatial scale. Discriminant analysis of principal components (DAPC) with 18,147 SNPs revealed a clear separation of distinct genetic clusters, while the eight polymorphic microsatellites showed much less resolution in delineating Australian, Indonesian and Vietnamese samples (Figure 4). Ten principal components and three discriminant functions were retained in both analyses, conserving $41.4 \%$ of the variation for the SNP data and $73.7 \%$ of the variation for the microsatellite data. Membership probabilities, interpreted as proximities of individuals to different clusters [48], showed that genome-wide SNP markers achieved unambiguous separation of all groups.

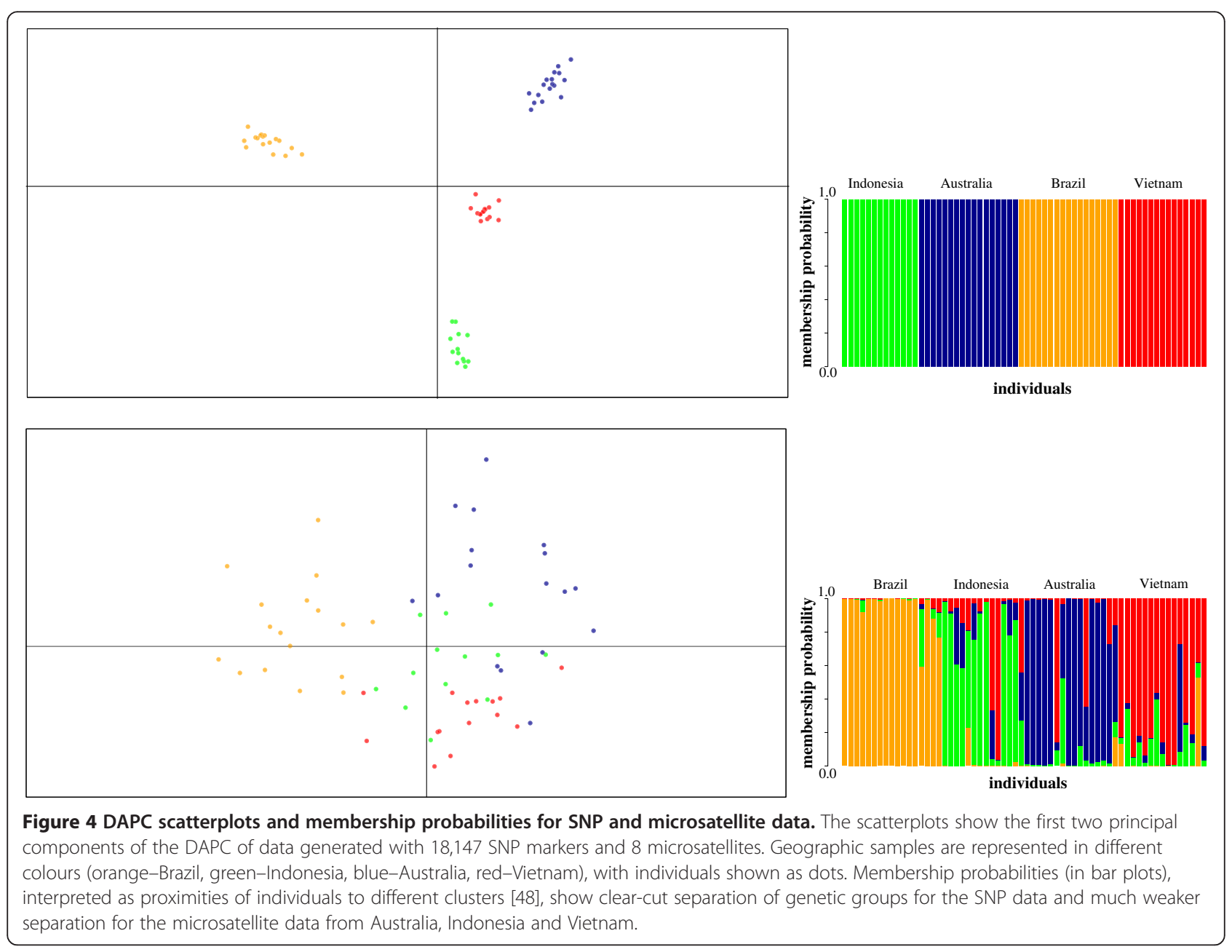


Using 1,504 RAD-generated SNPs, Brown et al. [29] recently provided strong evidence for the African ancestry of domesticated Ae. aegypti that spread throughout the (sub)tropical New World and from there, relatively recently, invaded Southeast Asia and the Pacific. Their set of SNPs enabled clear separation of African and neotropical populations, but provided no resolution within the Southeast Asia/Pacific group [29]. Here we showed that strong separation between populations from Vietnam, Indonesia and Australia is achieved with more markers.

Pair-wise $F_{\mathrm{ST}}$ values were larger for SNP markers than for microsatellites (Table 2), which is expected given the intrinsic mathematical dependence of $F_{\mathrm{ST}}$ on heterozygosity, number of alleles and their frequency [49]. Despite our small sample sizes, degree of differentiation for microsatellite markers was high and comparable to previous studies. For example, Gordonvale and Tri Nguyen collections had a pair-wise $F_{\mathrm{ST}}$ value of 0.093 in Endersby et al. [15] and 0.092 in our study.

\section{Fine scale relatedness}

934 SNPs showed remarkable power in distinguishing closely related individuals at a small spatial scale (Figure 5). Kinship coefficients $(k)$ and maximum likelihood relatedness $(r)$ were highly correlated $(r=0.884, p<0.01)$ and all imputed relationships had a strong support (likelihood ratio test $p<0.001)$. Six full-sib pairs and one half-sib pair were detected in the same trap (geographic distance of zero, Figure 5), while three half-sib pairs were found 420 meters apart. Conversely, only $19 \%$ of all putative relationships obtained with microsatellite markers were statistically supported. Microsatellite kinship coefficients were higher across spatial distances, but their log likelihood was not significantly greater than the log likelihood of the alternative relationships. The only supported related pair (full-sib, likelihood ratio test $p=0.013$ ) was found in the same trap (Figure 5).

The advantage of SNPs over microsatellites is increasingly reported in kinship and parentage analyses. Biallelic SNPs provide less information per locus, but this can be offset by their larger numbers. Unlike 17 microsatellites with low variability, a set of 960 SNPs ensured successful paternity and identity analysis in the European bison, Bison

Table 2 Estimates of pair-wise $F_{\mathrm{ST}}[60]$

\begin{tabular}{lcccc}
\hline & $\mathbf{B r}$ & $\mathbf{A u}$ & $\mathbf{I n}$ & $\mathbf{V i}$ \\
\hline $\mathrm{Br}$ & - & 0.146 & 0.108 & 0.177 \\
$\mathrm{Au}$ & 0.216 & - & 0.037 & 0.092 \\
$\mathrm{In}$ & 0.211 & 0.168 & - & 0.052 \\
$\mathrm{Vi}$ & 0.196 & 0.155 & 0.103 & - \\
\hline
\end{tabular}

$F_{\mathrm{ST}}$ values computed over all loci are reported for microsatellite markers (above diagonal) and genome-wide SNPs (below diagonal). bonasus [50]. In the wild sockeye salmon (Oncorhynchus nerka), 80 SNPs outperformed 11 highly variable microsatellites in parentage and kinship assignment [51]. On the other hand, using too many SNPs can lead to a decrease in power due to information redundancy from non-independent markers [21], but selecting a subset of approximately independent SNPs (as we did, choosing SNPs located on different supercontigs) can overcome this issue.

With high-resolution genetic data, analyses of ecological processes in Ae. aegypti can shift from traditional 'deme-based' methods to new individual-based methods. Assignment tests and similar approaches provide more direct inferences of dispersal and contemporary gene flow, avoiding a number of problems associated with traditional indirect inferences $[6,8]$. Fine scale mosquito movement and egg-laying patterns become tractable with numerous markers that reveal genetic differences even between highly related individuals. Identification of barriers or corridors for dispersal of this mosquito can be undertaken via individual-based landscape genetic analyses [52].

We have demonstrated that RAD sequencing provides insight into various population patterns and processes, going from broad-scale structuring, effective population size to fine scale relatedness. RAD-based markers therefore have the great potential to advance our understanding of mosquito ecology and assist in developing appropriate measures of vector control at local and regional scales.

\section{Conclusions}

Next-generation sequencing techniques such as RADseq provide an increasingly-affordable approach for generating numerous genetic markers to study disease vector populations. Here we developed a new set of bioinformatics tools that support the customization of restriction enzyme-based protocols for SNP discovery. We identified more than 18,000 putative SNPs in field samples of the major arbovirus vector Ae. aegypti. Our approach of using frequent cutting enzymes enabled unbiased sampling of genomic regions; we found $48 \%$ of variants in intergenic regions and $35 \%$ in exons and introns of over 2,300 genes. Their imputed effects in ORFs and UTRs were highly concordant with the effects found in the recent Ae. aegypti transcriptome sequencing study [43]. Our SNP set provided remarkable resolution in detecting broad-scale population structure and in estimating fine-scale relatedness. We demonstrated that a large SNP panel enables strong separation of Ae. aegypti populations even within a recently invaded neotropical region. Familial relatedness of samples collected from a small area could be confidently established with a subset of SNPs. RAD-based genetic data enables studies of basic 

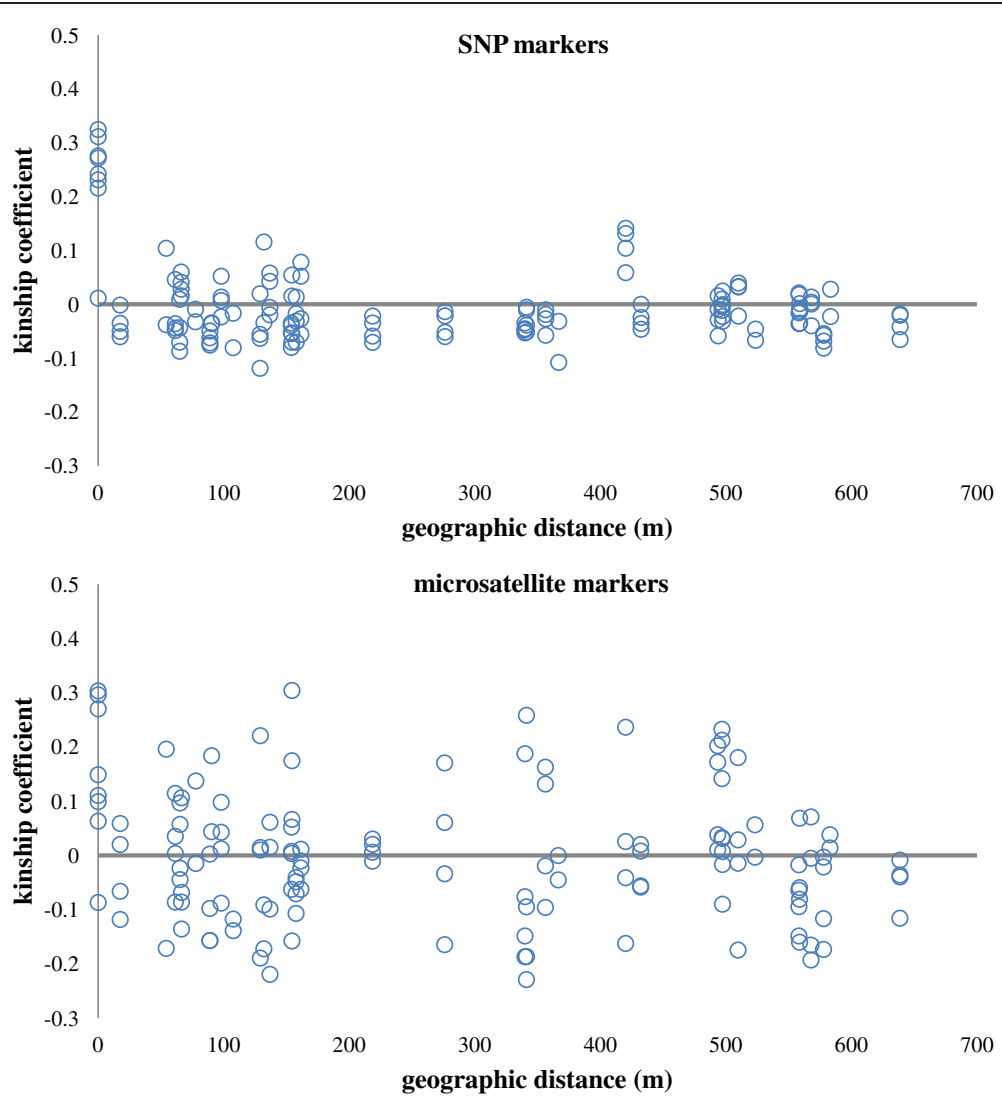

Figure 5 Kinship coefficients across geographic distances $(\mathbf{m})$. Loiselle's $k$ was estimated using 934 SNP markers and 8 microsatellite markers for pairs of individuals sampled within a $0.4 \mathrm{~km}^{2}$ area on the Paqueta Island, Rio de Janeiro, Brazil.

mosquito ecology and evolution in a versatile manner and at an extremely fine resolution, facilitating targeted control measures designed for a particular local mosquito population.

\section{Methods}

\section{Development of programs}

We developed a memory-efficient program for in silico genome digestion and calculation of potential ddRAD loci numbers. DDsilico is written in C language and is compiled for execution on Windows and Linux operating systems (see Additional file 1). DDsilico reads the input file with a multiple-sequence fasta format, such as concatenated (super) contig or chromosome sequences. It outputs a text file with a distribution of fragments for a given bin size $(1-n \mathrm{bp})$, distinguishing between sequenceable fragments (different overhang on each end) and nonsequenceable fragments (the same overhang on both ends). Also, DDsilico outputs the sum of nucleotides within each bin, corresponding to the fluorescence intensity in the Bioanalyzer assay, for comparison with the empirical digestion.

We also created a program for demultiplexing fastq files that accommodates various indexing schemes.
DDemux sorts reads for samples labelled with a single barcode or a combination of two barcodes that can vary in length (see Additional file 4). It was developed in C language and is compiled for execution on Windows and Ubuntu. DDemux has the capacity to sort reads for up to 125 samples in a single run.

\section{Sample collection}

Aedes aegypti larvae and adults were collected from water containers, ovitraps and BG traps at four locations: Paqueta island, Rio de Janeiro (Brazil), Yogyakarta (Indonesia), Hon Mieu island (Vietnam) and Gordonvale (Australia). No specific field ethics approval is needed for the collection of wild mosquitoes in these areas. Verbal consent was obtained from residents at each location where collections occurred on private property. These locations were not on protected land and the field collections did not involve endangered or protected species. Thirteen to 17 individuals were analyzed from each location (Table 1). GPS location was recorded for each collected individual. Samples were stored in $80 \%$ ethanol at $4^{\circ} \mathrm{C}$ until processing. Genomic DNA was extracted using Qiagen DNA Blood and Tissue kit (Venlo, Limburg, $\mathrm{NL}$ ), with the RNAse treatment step. 


\section{Double digest RAD library preparation}

$100 \mathrm{ng}$ of genomic DNA from each individual was digested in a $50 \mu \mathrm{L}$ reaction with 100 units each of NlaIII and $M l u C I$ restriction enzymes (New England Biolabs, Beverly MA, USA), NEB Buffer 4, BSA and water for $3 \mathrm{hrs}$ at $37^{\circ} \mathrm{C}$, without a heat kill step. The digestion products were cleaned with $1.5 \times$ volume of Ampure $\mathrm{XP}^{\mathrm{mix}}$ paramagnetic beads (Beckman Coulter, Brea, CA) and ligated to the modified Illumina P1 and P2 adapters. We used a combinatorial indexing scheme, labelling each individual with a unique combination of P1 and P2 barcodes containing variable length barcodes to increase library diversity at 5' and 3' ends (see Additional file 8). This way, 16 P1 and three P2 adapters allow for multiplexing of 48 individuals, while achieving a 2.5 -fold cost reduction for adapter generation. Forty $\mu \mathrm{L}$ ligation reactions were set up with $2 \mu \mathrm{L}$ of $2 \mu \mathrm{M}$ P1 and $6 \mu \mathrm{M}$ P2 adapters, 1000 units of T4 ligase and $1 \times \mathrm{T} 4$ buffer (New England Biolabs, Beverly MA, USA) and were incubated at $16^{\circ} \mathrm{C}$ overnight. Ligations were heat-inactivated at $65^{\circ} \mathrm{C}$ for $10 \mathrm{mi}-$ nutes and cooled down to a room temperature in a thermocycler at a rate of $1.5^{\circ} \mathrm{C}$ per 2 minutes. Adapterligated DNA fragments from all individuals were pooled and cleaned with $1.5 \times$ bead solution. Size selection of fragments between 300-450 bp was performed using a Pippin-Prep $2 \%$ gel cassette (Sage Sciences, Beverly, MA). Finally, $1 \mu \mathrm{L}$ of the size selected DNA was used as a template in a $10 \mu \mathrm{L}$ PCR reaction with $5 \mu \mathrm{L}$ of the Phusion High Fidelity $2 \times$ Master mix (New England Biolabs, Beverly MA, USA) and $2 \mu \mathrm{L}$ of $10 \mu \mathrm{M}$ P1 and P2 primers [28]. PCR conditions were: $98^{\circ} \mathrm{C}$ for $30 \mathrm{~s}$, 12 cycles of $98^{\circ} \mathrm{C}$ for $10 \mathrm{~s}, 60^{\circ} \mathrm{C}$ for $30 \mathrm{~s}, 72^{\circ} \mathrm{C}$ for $90 \mathrm{~s}$, and the final elongation at $72^{\circ} \mathrm{C}$ for $5 \mathrm{~min}$. Five such PCR reactions were pooled and cleaned with a $0.8 \times$ bead solution to make the final library. Two libraries were sequenced in two lanes of the Illumina HiSeq2000 platform to obtain 100 bp paired-end reads.

\section{Sequence processing and SNP calling}

To automate the process, we wrote bash scripts that take individually sorted raw sequences and output files ready for SNP calling (Figure 1, see Additional file 5). Initially, sequence quality scores were automatically converted of into the Sanger format. Sequences were then filtered with FASTX-Toolkit, trimming the reads to $80 \mathrm{bp}$ length and discarding all that have Phred score bellow 13. P1 and P2 reads were matched, and unpaired reads were sorted as orphans. Paired reads were aligned to the Ae. aegypti genome [53] using Bowtie version 0.12.7 [36]. Parameters for the un-gapped alignment included a maximum of three mismatches permitted in the seed, suppression of alignments if more than one reportable alignment exists, and a 'try-hard' option to find valid alignments. Orphans were then joined with all unaligned paired reads and single-end alignment was attempted. All aligned Bowtie output files were merged per individual and were imported into the Stacks pipeline.

A catalogue of RAD loci used for SNP discovery was created using the ref_map.pl pipeline in Stacks version 1.0 [37]. First, sequences aligned to the same genomic location were stacked together and merged to form loci. Here, only loci with a sequencing depth of five or more reads per individual were retained. SNPs at each locus were called using a maximum likelihood framework [54]. A catalogue was created of all possible loci and alleles and each individual was then matched against the catalogue. Finally, we used the program Populations in Stacks to process all the SNP data across individuals and calculate genome-wide measures of diversity, such as observed heterozygosity $\left(H_{\mathrm{O}}\right)$, expected heterozygosity $\left(H_{\mathrm{E}}\right)$ and nucleotide diversity $(\pi)$. We also estimated the longterm effective population sizes $\left(N_{\mathrm{e}}\right)$ using the nucleotide diversity averaged over all loci, where $\pi=4 * N_{\mathrm{e}}^{* *} \mu$ [55]. The mutation rate $(\mu)$ for SNPs is low, ranging between $10^{-8}$ and $10^{-9}$ per nucleotide site per generation [56].

To annotate and predict effects of filtered SNPs, we used SNPEff ver. $3.3 \mathrm{~h} \mathrm{[42]} \mathrm{with} \mathrm{default} \mathrm{settings} \mathrm{and}$ Ae. aegypti gene set AaegL1.4 (www.vectorbase.org/ organisms/aedes-aegypti/liverpool-lvp/AaegL1.4).

\section{Microsatellite genotyping}

We also screened all individuals at eight microsatellite loci (AG5, BbH08, BbA10, AC1, 470AG1, M201, 69TGA1, BbB19) described previously [10-12]. Primers were 'pigtailed' according to Brownstein et al. [57] and PCR products were directly labelled with fluorescent dye following the procedure by Blacket et al. [58]. Loci were separated into three multiplex $15 \mu \mathrm{L}$ reactions. The reaction mix was prepared according to the microsatellite amplification procedure in the QIAGEN ${ }^{\circ}$ Multiplex PCR Handbook and $0.5 \mathrm{ng}$ of DNA. The cycling protocol included: the initial incubation step at $95^{\circ} \mathrm{C}$ for 15 minutes, 35 amplification cycles with $94^{\circ} \mathrm{C}$ for $30 \mathrm{~s}, 60^{\circ} \mathrm{C}$ for $90 \mathrm{~s}$ and $72^{\circ} \mathrm{C}$ for $60 \mathrm{~s}$, followed by eight fluorescent labelling cycles with $94^{\circ} \mathrm{C}$ for $30 \mathrm{~s}, 53^{\circ} \mathrm{C}$ for $90 \mathrm{~s}$ and $72^{\circ} \mathrm{C}$ for $60 \mathrm{~s}$, and final extension at $60^{\circ} \mathrm{C}$ for 30 minutes. Sizing of PCR products was done with Applied Biosystems 3730 DNA Analyser with 500 LIZ size standard. GeneMarkerV2.2.0 (Softgenetics, State College, PA) was used for allele scoring.

\section{Testing the SNP markers against microsatellites for} detecting broad scale and fine-scale genetic patterns

We used Discriminant Analysis of Principal Components (DAPC) to identify and describe clusters of genetically related individuals implemented in the $\mathrm{R}$ package adegenet ver. 1.3-9.2 [48,59]. This multivariate method is suitable for analyzing large numbers of genome-wide SNPs, providing assignment of individuals to groups and a visual 
assessment of between-population differentiation. Because it does not rely on any particular population genetics model, DAPC is free of assumptions about HardyWeinberg equilibrium or linkage equilibrium [48].We therefore used a full set of 18,147 SNPs and eight microsatellites in this analysis. To avoid over-fitting of the discriminate functions, we retained ten principal components for both data sets. We also calculated Weir and Cockerhams's $F_{\mathrm{ST}}$ [60] in Genepop [61].

To explore the power of SNP and microsatellite markers to confidently assign relationships to pairs of individuals at a small spatial scale, we calculated Loiselle's kinship coefficients $k$ [62] for samples from Brazil in SPAGeDi [63]. First, in order to avoid strong linkage between SNPs, we made a subset of 934 markers by randomly sampling one SNP per supercontig. Then, as in Iacchei et al. [64], we considered Loiselle's coefficients $k$ to be between 0.25 and 0.375 for full-sibs and between 0.125 and 0.25 for half-sibs. A negative kinship coefficient indicates that a pair of individuals is less related than random pairs. We also calculated maximum likelihood relationship and used the likelihood ratio test with 1000 randomly simulated genotypes in ML-Relate [65].

\section{Availability of supporting data}

Sequencing data are deposited at NCBI's Sequence Read Archive (SRA) under the project accession number SRP040064 (http://www.ncbi.nlm.nih.gov/sra/?term=SRP04 0064). All other supporting data, including programs DDsilico and DDemux, are included as Additional files $1,2,3,4,5,6,7,8$.

\section{Additional files}

Additional file 1: The compressed archive contains $D D$ silico executables for Widows (ddsilico.exe) and Linux (ddsilico), the complete Stinger GFP sequence in fasta format (pstinger.fa) as a test input file, and README file (README.txt).

Additional file 2: Figure S2. Comparison of DDsilico and Bioanalyzer results for Stinger GFP. DNA of the transformation vector Stinger GFP was digested with restriction enzymes (N/alll and MluCl) and compared with DDsilico results. Please note that the Bioanalyzer profile has two additional peaks at $35 \mathrm{bp}$ and 10,380 bp that are the internal size standards for the High Sensitivity DNA chip (Agilent Technologies, Santa Clara, CA) (i.e. the two peaks are NOT part of the digested vector DNA). Also, Bioanalyzer peaks bellow $150 \mathrm{bp}$ were only partially retained with the paramagnetic bead solution during the required purification step. Table S2. Fragment size distribution from the Bioanalyzer and DDsilico runs. Concordance between the two results is high, with only two very low intensity peaks (88 bp and $323 \mathrm{bp}$ ) present in the Bioanalyzer but absent in DDsilico.

Additional file 3: Figure S3. DDsilico results for Aedes aegypti genome digested with various combinations of restriction enzymes. The $x$-axis represents fragment sizes (in base pairs), and the $y$-axis represent the number of fragments for a given size. Blue line depicts fragments that are not sequenceable (created by the same enzyme), while a red line depicts potential ddRAD loci. Distinguishing between amplifiable fragments is a useful DDsilico feature, as some double digestions produce numerous fragments in the desirable size range (100-500 bp), but only a small proportion constitutes potential ddRAD loci.

Additional file 4: The compressed archive contains DDemux executables for Widows (ddemux.exe) and Linux (ddemux), small Illumina fastq sequencing files (20000_R1.fq and 20000_R2.fq) as test input files, configuration file (config.txt) and README file (README.txt).

Additional file 5: Compressed bash scripts that take individually sorted raw sequences and output files ready for SNP calling. The compressed archive contains two scripts: preprocess_paired_reads.sh that also invokes sort_paired_reads.sh. preprocess_paired_reads.sh performs conversion of sequence quality score into the Sanger format, ensuring standardization of sequence data generated by various Illumina machines with different Q score set-ups. Sequences are then filtered with

FASTX-Toolkit, trimmed to a desired reads length and filtered based on the Phred score. After these processes, reads are sorted and matched as pairs, while orphans are kept in separate files (for $\mathrm{P} 1$ and $\mathrm{P} 2$ reads). Reads are then uniquely aligned in Bowtie, as described in Materials and Methods. The final output file contains concatenated uniquely aligned reads for each sample ready for the SNP calling pipeline.

Additional file 6: Source SNP and genotyping data in VCF. Compressed Variant calling format (VCF) file for a set of 18,147 SNPS filtered from the Stacks catalogue. Information field contains predicted SNP effects. Imputed individual genotypes from this file were used for the population genetic analyses. Additional file $6 \mathrm{~b}$ is a tab delimited file with SNP positions in the improved chromosome assembly by Juneja et al. [30], where the last field (ID) corresponds to the ID field in VCF.

Additional file 7: Table S6. Descriptive statistic for eight Aedes aegypti microsatellites. $N$ - number of individuals screened for a particular microsatellite locus, $\mathrm{Na}$ - number of alleles per locus, $\mathrm{Ho}$ - observed heterozygosity, He - expected heterozygosity, $F_{I S}$ - fixation index.

Additional file 8: Adapter and PCR primer sequences. We have modified the adapter sequences from [28] by incorporating variable length barcodes on both P1 and P2 adapters to increase the sequence diversity at $5^{\prime}$ and $3^{\prime}$ ends and to create a cost-effective barcoding scheme.

\section{Competing interests}

The authors declare that they have no competing interests.

\section{Authors' contributions}

AAH, ARW and GR conceived the study. GR designed and performed the RAD library constructions. IF and GR developed programs and performed bioinformatics analyses. GR analyzed data. GR and AAH drafted the manuscript. All authors have read and approved the final manuscript.

\section{Acknowledgements}

This work is funded by a grant from the National Health and Medical Research Council, Fellowship from the Australian Research Council Australia to $\mathrm{AAH}$, a grant from the Foundation for the National Institutes of Health through the Grand Challenges in Global Health Initiative of the Bill and Melinda Gates Foundation, and the Early Career Researcher Grant Scheme awarded to GR. Computational resources were provided by the NeCTAR Research Cloud and Edward HPC at the University of Melbourne. We are grateful to a number of researchers for providing field collections of Aedes aegypti: Renata Schama, Rafael Maceil-de Freitas and Luciano Moreira from FIOCRUZ for Brazilian samples; Hoang Le Nguyen from National Institute Of Hygiene And Epidemiology for Vietnamese samples; Eggi Arguni and Warsito Tantowijoyo from Universitas Gadjah Mada for Indonesian samples; and Brian Montgomery from Monash University and Scott Ritchie from James Cook University for Australian samples. We would also like to thank Philippa Griffin and two anonymous reviewers for the insightful comments on the earlier version of the manuscript. 


\section{References}

1. Bhatt S, Gething PW, Brady OJ, Messina JP, Farlow AW, Moyes CL, Drake JM, Brownstein JS, Hoen AG, Sankoh O, Myers MF, George DB, Jaenisch T, Wint GR, Simmons CP, Scott TW, Farrar JJ, Hay SI: The global distribution and burden of dengue. Nature 2013, 496(7446):504-507.

2. World Health Organization: Global Strategy for dengue prevention and control, 2012-2020. WHO report 2012 (reference number WHO/HTM/NTD/ VEM/2012.5). Geneva, Switzerland. Retrieved from http://www.who.int/ denguecontrol/9789241504034/en/.

3. Hoffmann AA, Montgomery BL, Popovici J, Iturbe-Ormaetxe I, Johnson PH, Muzzi F, Greenfield M, Durkan M, Leong YS, Dong Y, Cook H, Axford J, Callahan AG, Kenny N, Omodei C, McGraw EA, Ryan PA, Ritchie SA, Turelli M, O'Neill SL: Successful establishment of Wolbachia in Aedes populations to suppress dengue transmission. Nature 2011, 476(7361):454-457.

4. Barton NH, Turelli M: Spatial waves of advance with bistable dynamics: cytoplasmic and genetic analogues of Allee effects. Am Nat 2011, 178(3):E48-E75.

5. Yan G, Chadee DD, Severson DW: Evidence for genetic hitchiling effect associated with insecticide resistance in Aedes aegypti. Genetics 1998, 148:793-800.

6. Keyghobadi $\mathrm{N}$ : The genetic implications of habitat fragmentation for animals. Can J Zool 2007, 85(10):1049-1064.

7. Urdaneta-Marquez L, Failloux AB: Population genetic structure of Aedes aegypti, the principal vector of dengue viruses. Infect Genet Evol 2011, 11(2):253-261.

8. Anderson CD, Epperson BK, Fortin MJ, Holderegger R, James PM, Rosenberg MS, Scribner KT, Spear S: Considering spatial and temporal scale in landscape-genetic studies of gene flow. Mol Ecol 2010, 19(17):3565-3575.

9. Hlaing T, Tun-Lin W, Somboon P, Socheat D, Setha T, Min S, Chang MS, Walton C: Mitochondrial pseudogenes in the nuclear genome of Aedes aegypti mosquitoes: implications for past and future population genetic studies. BMC Genet 2009, 10:11.

10. Chambers EW, Meece JK, McGowan JA, Lovin DD, Hemme RR, Chadee DD, McAbee K, Brown SE, Knudson DL, Severson DW: Microsatellite isolation and linkage group identification in the yellow fever mosquito Aedes aegypti. J Hered 2007, 98(3):202-210.

11. Slotman MA, Kelly NB, Harrington LC, Kitthawee S, Jones JW, Scott TW, Caccone A, Powell JR: Polymorphic microsatellite markers for studies of Aedes aegypti (Diptera: Culicidae), the vector of dengue and yellow fever. Mol Ecol Notes 2007, 7(1):168-171.

12. Lovin DD, Washington KO, deBruyn B, Hemme RR, Mori A, Epstein SR, Harker BW, Streit TG, Severson DW: Genome-based polymorphic microsatellite development and validation in the mosquito Aedes aegypti and application to population genetics in Haiti. BMC Genomics 2009, 10:590.

13. Brown JE, McBride CS, Johnson P, Ritchie S, Paupy C, Bossin H, Lutomiah J, Fernandez-Salas I, Ponlawat A, Cornel AJ, Black WC, Gorrochotegui-Escalante N, Urdaneta-Marquez L, Sylla M, Slotman M, Murray KO, Walker C, Powell JR: Worldwide patterns of genetic differentiation imply multiple 'domestications' of Aedes aegypti, a major vector of human diseases. Proc Biol Sci 2011, 278(1717):2446-2454.

14. Hemme RR, Thomas CL, Chadee DD, Severson DW: Influence of urban landscapes on population dynamics in a short-distance migrant mosquito: evidence for the dengue vector Aedes aegypti. PloS Neglect Trop D 2010, 4(3):e634.

15. Endersby NM, Hoffmann AA, White VL, Lowenstein S, Ritchie S, Johnson PH, Rapley LP, Ryan PA, Nam VS, Yen NT, Kittiyapong P, Weeks AR: Genetic structure of Aedes aegypti in Australia and Vietnam revealed by microsatellite and exon primed intron crossing markers suggests feasibility of local control options. J Med Entomol 2009, 46(5):1074-1083.

16. Olanratmanee P, Kittayapong P, Chansang C, Hoffmann AA, Weeks AR, Endersby NM: Population genetic structure of Aedes (Stegomyia) aegypti (L.) at a micro-spatial scale in Thailand: implications for a dengue suppression strategy. PLoS Neglect Trop D 2013, 7(1):e1913.

17. Endersby NM, Hoffmann AA, White VL, Ritchie SA, Johnson PH, Weeks AR: Changes in the genetic structure of Aedes aegypti (Diptera: Culicidae) populations in Queensland, Australia, across two seasons: implications for potential mosquito releases. J Med Entomol 2011, 48(5):999-1007.

18. Reiter P, Amador MA, Anderson AR, Clark GG: Short Report: Dispersal of Aedes aegypti in an urban area after blood feeding as demonstrated by rubidium-marked eggs. Am J Trop Med Hyg 1995, 52(2):177-179.
19. Goncalves da Silva A, Cunha IC, Santos WS, Luz SL, Ribolla PE, Abad-Franch F: Gene flow networks among American Aedes aegypti populations. Evol Appl 2012, 5(7):664-676.

20. Rašić G, Endersby NM, Williams C, Hoffmann AA: Using Wolbachia-based release for suppression of Aedes mosquitoes: insights from genetic data and population simulations. Ecol Appl 2014. http://dx.doi.org/10.1890/13-1305.1

21. Santure AW, Stapley J, Ball AD, Birkhead TR, Burke T, Slate JON: On the use of large marker panels to estimate inbreeding and relatedness: empirical and simulation studies of a pedigreed zebra finch population typed at 771 SNPs. Mol Ecol 2010, 19(7):1439-1451.

22. Cariou M, Duret $L$, Charlat S: Is RAD-seq suitable for phylogenetic inference? An in silico assessment and optimization. Ecol Evol 2013, 3(4):846-852.

23. Nene V, Wortman JR, Lawson D, Haas B, Kodira C, Tu ZJ, Loftus B, Xi ZY, Megy K, Grabherr M, Ren QH, Zdobnov EM, Lobo NF, Campbell KS, Brown SE, Bonaldo MF, Zhu JS, Sinkins SP, Hogenkamp DG, Amedeo P, Arensburger P, Atkinson PW, Bidwell S, Biedler J, Birney E, Bruggner RV Costas J, Coy MR, Crabtree J, Crawford M, et al: Genome sequence of Aedes aegypti, a major arbovirus vector. Science 2007, 316(5832):1718-1723.

24. Baird NA, Etter PD, Atwood TS, Currey MC, Shiver AL, Lewis ZA, Selker EU, Cresko WA, Johnson EA: Rapid SNP discovery and genetic mapping using sequenced RAD markers. PLoS One 2008, 3(10):e3376.

25. Miller MR, Dunham JP, Amores A, Cresko WA, Johnson EA: Rapid and cost-effective polymorphism identification and genotyping using restriction site associated DNA (RAD) markers. Genome Res 2007, 17(2):240-248.

26. Davey JW, Hohenlohe PA, Etter PD, Boone JQ, Catchen JM, Blaxter ML: Genome-wide genetic marker discovery and genotyping using next-generation sequencing. Nat Rev Genet 2011, 12(7):499-510.

27. Etter PD, Bassham S, Hohenlohe PA, Johnson EA, Cresko WA: SNP discovery and genotyping for evolutionary genetics using RAD sequencing. Methods Mol Biol 2011, 772:157-178.

28. Peterson BK, Weber JN, Kay EH, Fisher HS, Hoekstra HE: Double digest RADseq: an inexpensive method for de novo SNP discovery and genotyping in model and non-model species. PLoS One 2012, 7(5):e37135.

29. Brown JE, Evans BR, Zheng W, Obas V, Barrera-Martinez L, Egizi A, Zhao H, Caccone A, Powell JR: Human impacts have shaped historical and recent evolution in Aedes aegypti, the dengue and yellow fever mosquito. Evolution 2014, 68(2):514-525.

30. Juneja P, Osei-Poku J, Ho YS, Ariani CV, Palmer WJ, Pain A, Jiggins FM: Assembly of the genome of the disease vector Aedes aegypti onto a genetic linkage map allows mapping of genes affecting disease transmission. PLoS Neglect Trop D 2014, 8(1):e2652

31. Elshire RJ, Glaubitz JC, Sun Q, Poland JA, Kawamoto K, Buckler ES, Mitchell SE: A robust, simple genotyping-by-sequencing (GBS) approach for high diversity species. PLoS One 2011, 6(5):e19379.

32. Poland JA, Brown PJ, Sorrells ME, Jannink JL: Development of high-density genetic maps for barley and wheat using a novel two-enzyme genotyping-by-sequencing approach. PLoS One 2012, 7(2):e32253.

33. Wang JW, Xia YD, Li LL, Gong DS, Yao Y, Luo HJ, Lu HL, Yi N, Wu HL, Zhang XQ Tao Q, Gao F: Double restriction-enzyme digestion improves the coverage and accuracy of genome-wide $\mathrm{CpG}$ methylation profiling by reduced representation bisulfite sequencing. BMC Genomics 2013, 14:11.

34. Toonen RJ, Puritz JB, Forsman ZH, Whitney JL, Fernandez-Silva I, Andrews KR: ezRAD: a simplified method for genomic genotyping in non-model organisms. Peer J 2013, 1:e203.

35. Stolle E, Moritz RF: RESTseq-efficient benchtop population genomics with RESTriction Fragment SEQuencing. PLoS One 2013, 8(5):e63960.

36. Langmead B, Trapnell C, Pop M, Salzberg SL: Ultrafast and memory-efficient alignment of short DNA sequences to the human genome. Genome Biol 2009, 10:R25.

37. Catchen J, Hohenlohe PA, Bassham S, Amores A, Cresko WA: Stacks: an analysis tool set for population genomics. Mol Ecol 2013, 22(11):3124-3140.

38. Minoche AE, Dohm JC, Himmelbauer H: Evaluation of genomic high-throughput sequencing data generated on Illumina HiSeq and genome analyzer systems. Genome Biol 2011, 12(11):R112.

39. Pujolar JM, Jacobsen MW, Frydenberg J, Als TD, Larsen PF, Maes GE, Zane L, Jian JB, Cheng L, Hansen MM: A resource of genome-wide single-nucleotide polymorphisms generated by RAD tag sequencing 
in the critically endangered European eel. Mol Ecol Resour 2013, 13(4):706-714.

40. Barchi L, Lanteri S, Portis E, Acquadro A, Vale G, Toppino L, Rotino GL: Identification of SNP and SSR markers in eggplant using RAD tag sequencing. BMC Genomics 2011, 12:304.

41. Van Bers NEM, Van Oers K, Kerstens HHD, Dibbits BW, Crooijmans RPMA, Visser ME, Groenen MAM: Genome-wide SNP detection in the great tit Parus major using high throughput sequencing. Mol Ecol 2010, 19:89-99.

42. Cingolani P, Platts A, le Wang L, Coon M, Nguyen T, Wang L, Land SJ, Lu X, Ruden DM: A program for annotating and predicting the effects of single nucleotide polymorphisms, SnpEff: SNPs in the genome of Drosophila melanogaster strain w1118; iso-2; iso-3. Fly 2012, 6(2):80-92.

43. Bonizzoni M, Britton M, Marinotti O, Dunn WA, Fass J, James AA: Probing functional polymorphisms in the dengue vector, Aedes aegypti. BMC Genomics 2013, 14:739.

44. Morlais I, Severson DW: Intraspecific DNA variation in nuclear genes of the mosquito Aedes aegypti. Insect Mol Biol 2003, 12(6):631-639.

45. Morin PA, Luikart G, Wayne RK, Grp SW: SNPs in ecology, evolution and conservation. Trends Ecol Evol 2004, 19(4):208-216.

46. Jeffery JAL, Yen NT, Nam VS, Nghia LT, Hoffmann AA, Kay BH, Ryan PA: Characterizing the Aedes aegypti population in a Vietnamese village in preparation for a Wolbachia-based mosquito control strategy to eliminate dengue. PLoS Neglect Trop D 2009, 3(11):e552.

47. Ritchie SA, Montgomery BL, Hoffmann AA: Novel estimates of Aedes aegypti (Diptera: Culicidae) population size and adult survival based on Wolbachia releases. J Med Entomol 2013, 50(3):624-631.

48. Jombart T, Devillard S, Balloux F: Discriminant analysis of principal components: a new method for the analysis of genetically structured populations. BMC Genet 2010, 11:94.

49. Jakobsson M, Edge MD, Rosenberg NA: The relationship between F(ST) and the frequency of the most frequent allele. Genetics 2013, 193(2):515-528.

50. Tokarska M, Marshall T, Kowalczyk R, Wojcik JM, Pertoldi C, Kristensen TN, Loeschcke V, Gregersen VR, Bendixen C: Effectiveness of microsatellite and SNP markers for parentage and identity analysis in species with low genetic diversity: the case of European bison. Heredity 2009, 103(4):326-332.

51. Hauser L, Baird M, Hilborn R, Seeb LW, Seeb JE: An empirical comparison of SNPs and microsatellites for parentage and kinship assignment in a wild sockeye salmon (Oncorhynchus nerka) population. Mol Ecol Resour 2011, 11(Suppl 1):150-161.

52. Storfer A, Murphy MA, Evans JS, Goldberg CS, Robinson S, Spear SF, Dezzani R, Delmelle E, Vierling L, Waits LP: Putting the "landscape" in landscape genetics. Heredity 2007, 98(3):128-142.

53. VectorBase: VectorBase. Adv Physiol Educ https://www.vectorbase.org/ organisms/aedes-aegypti/liverpool/aaegl1.

54. Hohenlohe PA, Bassham S, Etter PD, Stiffler N, Johnson EA, Cresko WA Population genomics of parallel adaptation in threespine stickleback using sequenced RAD tags. PloS Genet 2010, 6(2):e1000862.

55. Tajima F: Evolutionary relationship of DNA sequences in finite populations. Genetics 1983, 105:437-460.

56. Brumfield RT, Beerli P, Nickerson DA, Edwards SV: The utility of single nucleotide polymorphisms in inferences of population history. Trends Ecol Evol 2003, 18(5):249-256.

57. Brownstein MJ, Carpten JD, Smith JR: Modulation of non-templated nucleotide addition by tag DNA polymerase: Primer modifications that facilitate genotyping. Biotechniques 1996, 20(6):1004-1006. 1008-1010.

58. Blacket MJ, Robin C, Good RT, Lee SF, Miller AD: Universal primers for fluorescent labelling of PCR fragments - an efficient and cost-effective approach to genotyping by fluorescence. Mol Ecol Resour 2012, 12(3):456-463.

59. Jombart T, Ahmed I: adegenet 1.3-1: new tools for the analysis of genome-wide SNP data. Bioinformatics 2011, 27(21):3070-3071.

60. Weir BS, Cockerham CC: Estimating F-statistics for the analysis of population structure. Evolution 1984, 38(6):1358-1370.

61. Raymond M, Rousset F: GENEPOP (Version 1.2): population genetics software for exact tests and ecumenicism. J Hered 1995, 86(3):248-249.

62. Loiselle BA, Sork VL, Nason J, Graham C: Spatial genetic-structure of a tropical understory shrub, Psychotria officinalis (Rubiaceae). Am J Bot 1995, 82(11):1420-1425.
63. Hardy OJ, Vekemans $X$ : Spagedi: a versatile computer program to analyse spatial genetic structure at the individual or population levels. Mol Ecol Notes 2002, 2(4):618-620.

64. lacchei M, Ben-Horin T, Selkoe KA, Bird CE, Garcia-Rodriguez FJ, Toonen RJ: Combined analyses of kinship and $\mathrm{F}_{\mathrm{ST}}$ suggest potential drivers of chaotic genetic patchiness in high gene-flow populations. Mol Ecol 2013, 22(13):3476-3494.

65. Kalinowski ST, Wagner AP, Taper ML: ML-RELATE: a computer program for maximum likelihood estimation of relatedness and relationship. Mol Ecol Notes 2006, 6(2):576-579.

doi:10.1186/1471-2164-15-275

Cite this article as: Rašić et al.: Genome-wide SNPs lead to strong signals of geographic structure and relatedness patterns in the major arbovirus vector, Aedes aegypti. BMC Genomics 2014 15:275.

\section{Submit your next manuscript to BioMed Central and take full advantage of:}

- Convenient online submission

- Thorough peer review

- No space constraints or color figure charges

- Immediate publication on acceptance

- Inclusion in PubMed, CAS, Scopus and Google Scholar

- Research which is freely available for redistribution 